\title{
Regulation and role of hormone-sensitive lipase in rat skeletal muscle
}

\author{
Morten Donsmark ${ }^{1 *}$, Jozef Langfort ${ }^{2}$, Cecilia Holm ${ }^{3}$, Thorkil Ploug ${ }^{1}$ and Henrik Galbo ${ }^{1,4}$ \\ ${ }^{1}$ Copenhagen Muscle Research Centre, Department of Medical Physiology, The Panum Institute, University of Copenhagen, \\ Blegdamsvej 3, 2200 Copenhagen N, Denmark \\ ${ }^{2}$ Laboratory of Experimental Pharmacology, The Polish Academy of Sciences, Warsaw, Poland \\ ${ }^{3}$ Section for Molecular Signalling, Department of Cell and Molecular Biology, Lund University, Sweden \\ ${ }^{4}$ Department of Rheumatology, Bispebjerg Hospital, Copenhagen, Denmark
}

\begin{abstract}
Intramyocellular triacylglycerol (TG) is an important energy store, and the energy content of this depot is higher than the energy content of the muscle glycogen depot. It has recently been shown that the mobilization of fatty acids from this TG pool may be regulated by the neutral lipase hormone-sensitive lipase (HSL). This enzyme is known to be rate limiting for intracellular TG hydrolysis in adipose tissue. The presence of HSL has been demonstrated in all muscle fibre types by Western blotting of muscle fibres isolated by collagenase treatment or after freeze-drying. The content of HSL varies between fibre types, being higher in oxidative fibres than in glycolytic fibres. When analysed under conditions optimal for HSL, neutral lipase activity in muscle can be stimulated by adrenaline as well as by contractions. These increases are abolished by the presence of anti-HSL antibody during analysis. Moreover, immunoprecipitation with affinity-purified anti-HSL antibody causes similar reductions in muscle HSL protein concentration and in measured neutral lipase responses to contractions. The immunoreactive HSL in muscle is stimulated by adrenaline via $\beta$-adrenergic activation of cAMPdependent protein kinase (PKA). From findings in adipocytes it is likely that PKA phosphorylates HSL at residues $\mathrm{Ser}^{563}, \mathrm{Ser}^{659}$ and $\mathrm{Ser}^{660}$. Contraction probably also enhances muscle HSL activity by phosphorylation, because the contraction-induced increase in HSL activity is elevated by the protein phosphatase inhibitor okadaic acid and reversed by alkaline phosphatase. A novel signalling pathway in muscle by which HSL activity may be stimulated by protein kinase $\mathrm{C}$ (PKC) via extracellular signal-regulated kinase (ERK) has been demonstrated. In contrast to previous findings in adipocytes, in muscle the activation of ERK is not necessary for stimulation of HSL by adrenaline. However, contraction-induced HSL activation is mediated by PKC, at least partly via the ERK pathway. In fat cells ERK is known to phosphorylate HSL at $\mathrm{Ser}^{600}$. Hence, phosphorylation of different sites may explain the finding that in muscle the effects of contractions and adrenaline on HSL activity are partially additive. In line with the view that the two stimuli act by different mechanisms, training increases contraction-mediated HSL activation but diminishes adrenaline-mediated HSL activation in muscle. In conclusion, HSL is present in skeletal muscle and can be activated by phosphorylation in response to both adrenaline and muscle contractions. Training increases contraction-mediated HSL activation, but decreases adrenaline-mediated HSL activation in muscle.
\end{abstract}

Hormone-sensitive lipase: Adrenaline: Extracellular signal-regulated kinase: Exercise: Muscle fibre types

The enzyme hormone-sensitive lipase (HSL) was first identified in adipose tissue as a lipase sensitive to catecholamines by Vaughan et al. (1964). It is now well established that HSL is the rate-limiting enzyme of intracellular triacylglycerol (TG) breakdown in adipose tissue
(Holm et al. 2000). Interestingly, from recent studies on skeletal muscle it seems probable that HSL also plays a key role in the regulation of intramuscular TG breakdown (Langfort et al. 1999, 2000, 2002, 2003; Enevoldsen et al. 2001; Donsmark et al. 2003).

\footnotetext{
Abbreviations: AICAR, 5-aminoimidazole-4-carboxamide-1- $\beta$-D-riboside; AMPK, AMP-activated kinase; DG, diacylglycerols; ERK, extracellular signalregulated kinase; HSL, hormone-sensitive lipase; PKA, cAMP-dependent protein kinase; PKC, protein kinase C; TG, triacylglycerols. *Corresponding author: Dr Morten Donsmark, fax +45 35327555, email mdonsmark@mfi.ku.dk
} 
In skeletal muscle a considerable amount of $\mathrm{TG}$ is stored, and this store contains more energy than the muscle glycogen depot (Van der Vusse \& Reneman, 1996). The stored TG is deposited in lipid droplets that are localized in close proximity to the mitochondria where the $\beta$ oxidation of fatty acids takes place (Oscai et al. 1990). Several studies have demonstrated that the intramuscular TG constitutes a dynamic energy store that can be mobilized by catecholamines (Abumrad et al. 1980; Fredrikson et al. 1986), exercise (Carlson et al. 1971; Reitman et al. 1973) and electrical stimulation of skeletal muscle (Spriet et al. 1986; Hopp \& Palmer, 1990). The breakdown of intramuscular TG by catecholamines is compatible with a role for HSL in the regulation of TG in skeletal muscle. However, three different types of TG lipases have been demonstrated in skeletal muscle, which are characterized by distinct $\mathrm{pH}$ optima (Oscai et al. 1990; Van der Vusse \& Reneman, 1996). One of these lipases is a lysosomal lipase with an in vitro $\mathrm{pH}$ optimum of 5. Another lipase is lipoprotein lipase, which has been accepted as the lipase responsible for intramuscular TG hydrolysis for several years because there is a direct relationship between lipoprotein lipase activity and TG breakdown under various conditions (Oscai et al. 1990). However, this notion is probably not correct because in muscle cells lipoprotein lipase is produced as a secretory protein and resides inside vesicles. Furthermore, the intracellular milieu would not be compatible with optimum functioning of lipoprotein lipase, which requires a $\mathrm{pH}$ of 8.5 and the presence of apoprotein C-2 (Oscai et al. 1990; Van der Vusse \& Reneman, 1996). The third type of lipase in muscle is a neutral lipase.

As mentioned earlier, in adipocytes the hydrolysis of TG is catalysed by HSL, which is a neutral lipase with a $\mathrm{pH}$ optimum of 7 . Interestingly, two studies have indicated by the use of Northern and Western blotting that HSL is expressed in skeletal muscle (Holm et al. 1987, 1988). However, it cannot be excluded that the HSL detected in these two studies originated from adipocytes interlaced between muscle fibres. Nevertheless, a possible role for HSL in skeletal muscle is in accordance with the previously mentioned influence of different physiological conditions on muscle TG metabolism. The present paper describes recent findings relating to HSL metabolism in rat muscle.

\section{Enzymic properties of hormone-sensitive lipase}

The breakdown of stored TG in adipocytes is catalyzed by the two enzymes HSL and monoacylglycerol lipase and takes place through three successive steps (Fredrikson et al. 1986; Straalfors et al. 1987). The breakdown of TG in adipocytes is controlled by HSL because in the nonstimulated state the enzyme has a ten-fold higher activity towards diacylglycerol (DG) than towards TG (Straalfors et al. 1987). When stimulated by catecholamines HSL becomes phosphorylated and activated by cAMP-dependent protein kinase (PKA) acting at residues $\operatorname{Ser}^{563}, \operatorname{Ser}^{659}$ and $\mathrm{Ser}^{660}$, the two latter sites being the major sites controlling activity and translocation (Anthonsen et al. 1998; Su et al. 2003). The catalytic activity is increased towards TG whereas the activity towards DG remains unchanged
(Straalfors et al. 1987; Yeaman, 1990; Anthonsen et al. 1998; Holm \& Østerlund, 1998). Thus, the activated form of HSL can be estimated by a TG substrate (HSL (TG) activity) and the total enzyme concentration can be measured using a DG substrate (HSL (DG) activity).

\section{Expression of hormone-sensitive lipase in rat skeletal muscle}

The expression of HSL in rat skeletal muscle was first demonstrated by Holm and colleagues using Western blotting (Holm et al. 1987) and Northern blotting (Holm et al. 1988). Both studies were carried out using non-dissected muscle tissue, which means that the detected HSL may have originated from adipocytes interlaced between muscle fibres. To exclude the possibility of contamination studies have been carried out on soleus muscles from young male Wistar rats weighing about $70 \mathrm{~g}$ to obtain pure muscle. In these muscle cells there is an abundance of lipid droplets scattered throughout the cytoplasm (Langfort et al. 1999). The presence of HSL in skeletal muscle cells has been demonstrated by Western blotting using an affinity-purified chicken antibody raised against HSL from rat adipose tissue. Expression of HSL has been found whether Western blotting is carried out on muscle fibres isolated by dissection after freeze-drying or on fibres washed three times after collagenase isolation (Langfort et al. 1999). Another finding that strengthens the notion that HSL is present inside skeletal muscle cells and not in surrounding fat cells is that HSL (TG):HSL (DG) in skeletal muscles differs from that in epididymal adipose tissue (Langfort et al. 1999). There is a correlation between the expression of HSL and fibre type; HSL expression is 4-9 times higher in soleus and diaphragm muscles, which contain predominantly type I and type IIa fibres, compared with extensor digitorum longus, which contains an equal number of type IIa and type IIb fibres, and epitrochlearis muscle, which mainly contains type IIb fibres (Langfort et al. 1999). There is a direct correlation between the variation in the expression of HSL in different muscle types and the known variation in TG concentration and oxidative capacity (Van der Vusse \& Reneman, 1996; Langfort et al. 1999).

\section{Activation of hormone-sensitive lipase by adrenaline in rat muscle}

HSL in rat skeletal muscle can be activated by adrenaline (Langfort et al. 1999). HSL (TG) activity increases after 2 min of stimulation with adrenaline and remains constant up to $20 \mathrm{~min}$. HSL (DG) activity does not change. This finding indicates that a neutral lipase in rat soleus muscle is activated by adrenaline and that the total enzyme concentration remains constant. Furthermore, the notion that HSL is the lipase that is responsible for the adrenalineinduced increase in HSL (TG) activity is confirmed, since no activation is found when the supernatant fraction from adrenaline-stimulated muscle is incubated with an antibody against HSL before measurements. Under identical conditions HSL (DG) activity is not completely abolished, 
indicating that other lipases are also expressed in rat skeletal muscle (Langfort et al. 1999).

On the basis of findings on the regulation of HSL in adipose tissue and heart it has been assumed that the effect of adrenaline on HSL in rat skeletal muscle is mediated by $\beta$-adrenergic receptors and PKA (Small et al. 1989; Langin et al. 1996; Anthonsen et al. 1998). This view is supported by the finding that the effect of adrenaline on HSL activation in muscle is completely prevented by the $\beta$-adrenergic receptor blocker, propranolol, and mimicked by incubation of the crude supernatant fraction from non-stimulated muscles with the catalytic subunit of PKA (Langfort et al. 1999). From findings in adipocytes it is likely that PKA phosphorylates HSL at residues $\mathrm{Ser}^{563}, \mathrm{Ser}^{659}$ and $\mathrm{Ser}^{660}$ (Anthonsen et al. 1998).

\section{Activation of hormone-sensitive lipase by muscle contractions}

In rat skeletal muscle HSL can be activated by electricallyinduced muscle contractions (Langfort et al. 2000). HSL (TG) activity increases markedly to the level observed during adrenaline stimulation (about $50 \%$ ) after $1 \mathrm{~min}$ of contractions and remains elevated for $5 \mathrm{~min}$. The activation of HSL is transient as HSL (TG) activity declines to the basal level during further stimulation. HSL (DG) activity does not change during muscle contractions (Langfort et al. 2000). These results indicate that the HSL enzyme activity increases whereas the HSL concentration remains constant during contractions. In confirmation that HSL is in fact the lipase responsible for the increase in neutral lipase activity associated with contraction, it has been shown that there is no measurable contraction-induced activation when the supernatant fraction from contracted muscle is incubated with anti-HSL antibody before measurements. Furthermore, immunoprecipitation of HSL causes an $80 \%$ reduction in HSL protein from the muscle supernatant fraction and this reduction is accompanied by similar reductions in both HSL (TG) activity and in the contraction-induced increase in HSL activity, again supporting the notion that HSL is the lipase responsible for the increase in neutral lipase activity during electrical stimulation of muscle (Langfort et al. 2000).

It may be speculated that the effect of electrical stimulation, rather than reflecting effects of contraction, is the result of activation of intramuscular sympathetic nerve fibres, which in turn increases the HSL activity in adipocytes or muscle cells. However, this reasoning is not correct, because the increase in HSL (TG) activity upon electrical stimulation is not affected by propranolol in a dose known to abolish the adrenaline-induced increase in HSL activity in muscle (Langfort et al. 2000). Furthermore, the response to electrical stimulation is intact in sympathectomized muscle (Langfort et al. 2000). The possibility that electrical stimulation directly activates adipocytes within muscle is excluded by the finding that electrical stimulation of epididymal fat for 5 min does not influence HSL (TG) activity in epididymal fat (Langfort et al. 2000). The involvement of phosphorylation in the contraction-induced activation of HSL has been investigated by incubating soleus muscles with the phosphatase inhibitor okadaic acid. The presence of the inhibitor doubles the contraction-mediated increase in HSL (TG) activity. Conversely, the contraction-induced increase in HSL (TG) activity is rapidly reversed by the addition of alkaline phosphatase to a homogenate from contracted muscle (Langfort et al. 2000). Thus, these findings indicate that the contraction-induced activation of HSL involves phosporylation and is independent of sympathetic activity.

In order to explore the interactions between adrenaline and contractions both stimuli have been applied simultaneously on incubated soleus and extensor digitorum longus muscles. The two stimuli have a partially additive effect on HSL (TG) activity in both muscle types (Langfort et al. 2003). This finding indicates that adrenaline and contractions activate HSL by at least partly different signalling pathways. It may be suggested that two different isoforms of HSL are expressed in skeletal muscle; one isoform being activated by adrenaline and the other activated by contractions. However, this suggestion is probably not correct, as full additivity would then be expected and only partial additivity is observed when the two stimuli are combined. Thus, the additivity observed at combined stimulation may reflect the possibility that adrenaline and contractions activate different kinases, which in turn phosphorylate HSL at different sites on the isoform expressed in skeletal muscle.

Based on the earlier information and the fact that the intramuscular $\mathrm{Ca}^{2+}$ concentration increases at the onset of muscle contractions it is tempting to speculate that the contraction-induced activation of HSL is mediated by a $\mathrm{Ca}^{2+}$-dependent mechanism. From studies of adipocytes it is known that the $\mathrm{Ca}^{2+}$ /calmodulin-dependent kinase II inhibits rather than increases the HSL activity (Garton et al. 1989). However, it has been suggested that $\mathrm{Ca}^{2+}$ stimulates HSL via $\mathrm{Ca}^{2+}$-activated protein kinase $\mathrm{C}$ (PKC). To test this hypothesis soleus muscle has been incubated with the PKC inhibitors Bisindolylmaleimide I and Calphostin C in concentrations that do not influence force development during muscle contractions. Both inhibitors abolish the contraction-mediated HSL activation but do not affect the adrenaline-mediated HSL activation (Fig. 1, only results from experiments with Calphostin $\mathrm{C}$ are shown; Donsmark et al. 2003). These findings indicate that PKC plays a key role in the contraction-mediated HSL activation. In line with this view it has recently been reported that in adipocytes PKC phosphorylates HSL at $\operatorname{Ser}^{600}$ via the extracellular signal-regulated kinase (ERK) (Greenberg et al. 2001). Moreover, contractions increase ERK activity in skeletal muscle, while adrenaline does not influence this activity (Napoli et al. 1998; Widegren et al. 2001). As Bisindolylmaleimide I inhibits all classes of PKC, whereas Calphostin $\mathrm{C}$ does not inhibit atypical PKC, either conventional or novel PKC isoforms seem to be involved in the contraction-induced HSL activation.

When incubated soleus muscle is treated with the specific mitogen-activated protein kinase kinase inhibitor U0126 the phosphorylation of ERK 1 and ERK 2 in response to contraction is totally abolished and is accompanied by a $50 \%$ inhibition of the contraction-mediated HSL activation. There is no effect on the adrenalinemediated HSL activation (Donsmark et al. 2003). These 
(a)

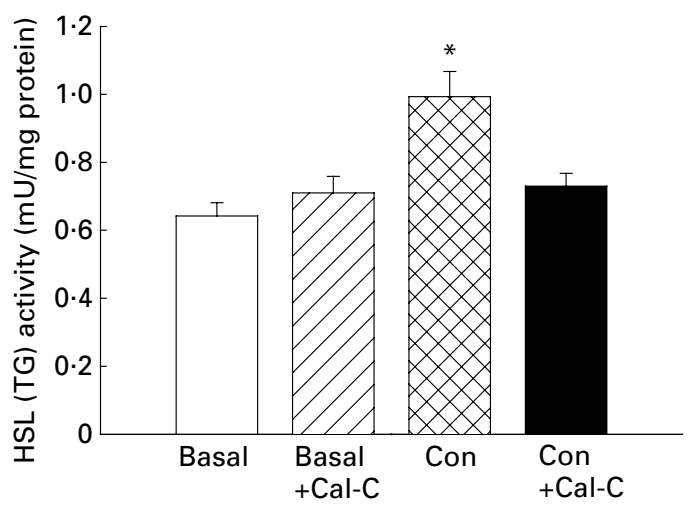

(b)

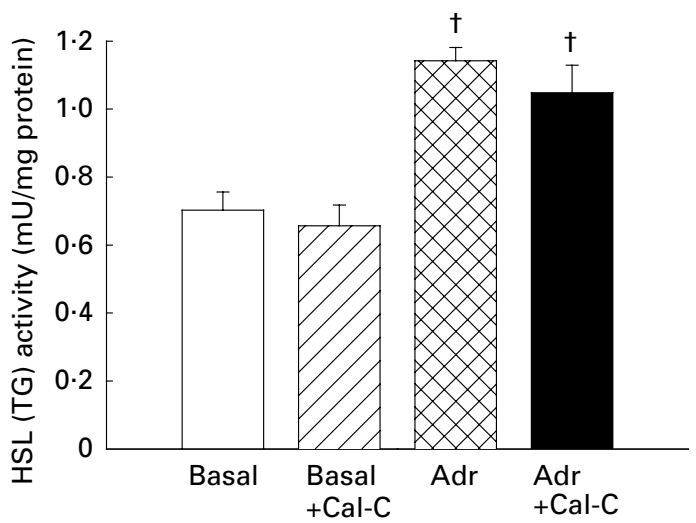

Fig. 1. Calphostin $C(\mathrm{Cal}-\mathrm{C})$ selectively inhibits the contractioninduced increase in hormone-sensitive lipase (HSL (triacylglycerol (TG))) activity. Soleus muscles were incubated in presence $(+\mathrm{Cal}-\mathrm{C})$ or absence of $2.5 \mu \mathrm{m}-\mathrm{Cal}-\mathrm{C}$ for $35 \mathrm{~min}$. Muscles were either rested (basal), or stimulated electrically to perform repeated tetanic contractions (Con; a) or stimulated with $5.5 \mu \mathrm{m}$-adrenaline (Adr; b) for the last $5 \mathrm{~min}$ of the incubation. Three muscles were used for each analysis, the two muscles from a given rat being both stimulated or rested and one incubated with $2.5 \mu \mathrm{m}-\mathrm{Cal}-\mathrm{C}$ and the other incubated without Cal-C. The muscles were freeze clamped and analysed for HSL (TG) activities. Values are means with their standard errors represented by vertical bars for three to nine analyses in each group. The mean value was significantly different from those for the other treatments: ${ }^{*} P<0.05$. The mean values were significantly different from those for the other treatment groups: $\dagger P<0.05$.

results indicate that ERK is involved in the contractionmediated HSL activation, and also that the effect of PKC on HSL is not mediated entirely by ERK. These results are supported by the finding that activated ERK mimics the effect of contractions when added to supernatant fraction from non-stimulated muscle, but has no effect when added to the supernantant fraction from electrically-stimulated muscle (Donsmark et al. 2003; Fig. 2). The view that PKC and ERK have a key position in the contraction-induced HSL activation has been further substantiated by the finding that the DG analogue phorbol 12-myristate 13-acetate activates ERK 1 and ERK 2 to the same extent as contraction and also increases HSL (TG) activity (Donsmark et al. 2003). However, the increase in HSL (TG) activity is only $50 \%$ of that obtained in response to contractions.

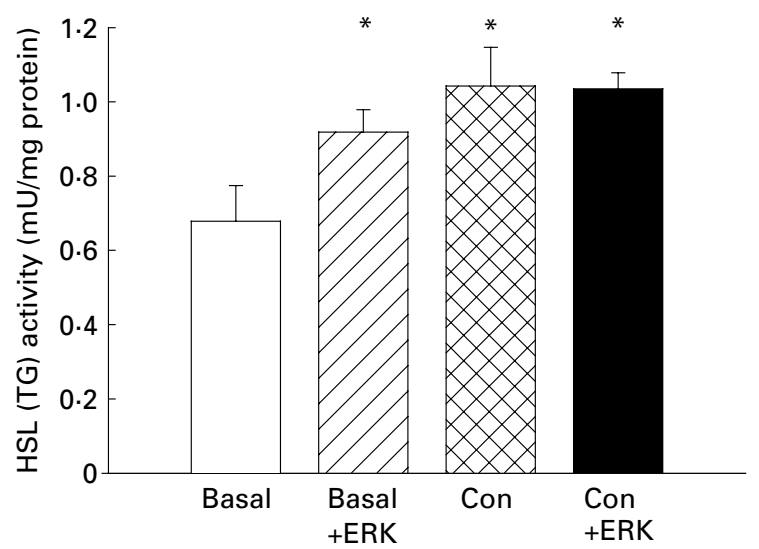

Fig. 2. Extracellular signal-regulated kinase (ERK) activates hormone-sensitive lipase (HSL (triacylglycerol (TG))) in crude supernatant fractions from rested (basal) but not from electricallystimulated muscle (5 min of repeated tetanic contractions; Con). Crude supernatant fractions from three rested or three electricallystimulated soleus muscles were incubated with or without activated ERK. Values are means with their standard errors represented by vertical bars for four to six incubations. Mean values were significantly different from that for the basal group: ${ }^{\star} P<0.05$.

These results again indicate that ERK activation only partially explains contraction-induced HSL activation. Finally, more indirect evidence that $\mathrm{Ca}^{2+}$ may be involved in the stimulation of HSL has been obtained by incubating soleus muscle with caffeine at a concentration known to increase the sarcoplasmic $\mathrm{Ca}^{2+}$ concentration without eliciting contraction. Caffeine causes a small (17\%) increase in HSL activity with no change in ERK activity (Donsmark et al. 2003). This finding is in line with the studies mentioned earlier that support the notion that during contractions PKC may also activate HSL by mechanisms other than through ERK.

\section{Influence of the AMP-activated kinase on hormone-sensitive lipase}

To further explore the regulation of HSL in rat skeletal muscle the influence of the AMP-activated kinase (AMPK which is known to enhance NEFA oxidation in skeletal muscle) on HSL activity has been investigated during basal conditions and during contractions. When epitrochlearis muscles are incubated with or without the AMP analogue and AMPK activator 5-aminoimidazole-4-carboxamide1- $\beta$-D-riboside (AICAR; $2 \mathrm{~mm}$ ), and either rested or contracted, HSL activity in resting muscle is markedly decreased by AICAR. In contrast, AICAR has no effect on the contraction-induced HSL activation. Pre-incubation of supernatant fractions with anti-HSL antibody completely prevents the AICAR-induced decrease in HSL (TG) activity, confirming that HSL is the neutral lipase that is manipulated by AICAR in rat muscle (M Donsmark, J Langfort, C Holm, T Ploug and $\mathrm{H}$ Galbo, unpublished results). These findings suggest that during intracellular energy crises in the resting state AMPK activity tends to preserve intramuscular TG stores by lowering HSL activity, thereby switching to aerobic and anaerobic glycolysis, 
which gives a higher ATP yield per molecule $\mathrm{O}_{2}$ combusted. During intense contractions AMPK activity in muscle is increased. This finding may explain why AICAR does not influence HSL (TG) activity during contractions: The increase in HSL (TG) activity during intense contractions is the net result of enhancing $\mathrm{Ca}^{2+}$-mediated mechanisms and inhibiting AMPK-mediated mechanisms, and any further AICAR-induced increase in AMPK may not be able to influence this balance.

\section{Role of hormone-sensitive lipase in skeletal muscle}

The effect of HSL on intramuscular TG breakdown has recently been investigated by using soleus muscle from HSL null mice. During muscle contractions there is an increase in glycerol release in wild type mice $(P<0 \cdot 056)$, but no change in the HSL null mice (M Donsmark, J Langfort, C Holm, T Ploug and H Galbo, unpublished results). These data are the first to indicate that HSL is responsible for the breakdown of intramuscular TG during muscle contraction.

\section{Effect of endurance training on hormone-sensitive lipase in rat skeletal muscle}

Long-term adaptations of HSL take place in adipose tissue during fasting, obesity, pregnancy and exercise training (Sztalryd \& Kraemer, 1994; Martin, 1996; Large et al. 1998, 1999; Enevoldsen et al. 2001). The influence of training on TG breakdown in exercising muscle is a controversial issue. However, most evidence indicates that in response to a single exercise bout at a given intensity there is a greater depletion of TG in trained muscle than in untrained muscle despite reduced catecholamine levels and plasma NEFA turnover (Galbo, 1983; Hurley et al. 1986; Phillips et al. 1996) . This response may reflect an increase in the amount of HSL in trained muscle or increased sensitivity to stimulation of the enzyme by adrenaline and/or contraction. It has previously been found that HSL protein concentration and total enzyme activity (HSL (DG) activity) in muscle do not differ between endurance-trained and sedentary rats (Enevoldsen et al. 2001). Furthermore, HSL sensitivity to stimulation by adrenaline is decreased in extensor digitorum longus and soleus muscles after training. The diminished HSL (TG): HSL (DG) during stimulation of trained muscle is accompanied by lower muscle glycerol release in vivo in trained rats compared with untrained rats (Enevoldsen $e t$ al. 2000). Thus, the difference in adrenaline activation of HSL cannot explain the greater muscle TG breakdown in trained rats compared with untrained rats. On the other hand, endurance training increases the contraction-mediated HSL (TG) activity in soleus muscle both in the absence and in the presence of adrenaline during contractions. Thus, an augmented muscle HSL (TG) response to exercise probably contributes to the training-induced enhancement of muscle TG breakdown during exercise (Langfort et al. 2002). No effect of training has been observed in extensor digitorum longus muscle. Assuming that this muscle is only minimally activated during the training (running) programme, the findings indicate that training enhances HSL activation in muscle by local contraction-mediated mechanisms, and not by systemic mechanisms. However, the different responses in soleus muscle compared with extensor digitorum longus muscle may also reflect differences in the fibre type composition between these muscles. Again, the opposite effects of training on contraction-mediated and adrenalinemediated HSL activation in muscle are in line with the view that the two stimuli act by different mechanisms.

\section{Conclusions}

In conclusion, HSL is present in skeletal muscle cells and is expressed at a higher level in oxidative fibres than in glycolytic fibres. The two physiological stimuli adrenaline and contractions increase muscle HSL activity, and the effects are partially additive. Adrenaline acts via $\beta$ adrenergic activation of PKA, while the effect of contractions is mediated by PKC, at least partly via the ERK pathway. Endurance training diminishes the sensitivity of muscle HSL to adrenaline but increases the contractionmediated HSL activation.

\section{Acknowledgements}

The studies were supported by grants from Novo Nordic Foundation, the Danish National Research Foundation (grant 504-14), the Danish Diabetes Foundation, The Danish Sports Research Council, the Swedish Research Council (grant no. 11284) and the Swedish Diabetes Association.

\section{References}

Abumrad NA, Tepperman HM \& Tepperman J (1980) Control of endogenous triglyceride breakdown in the mouse diaphragm. Journal of Lipid Research 21, 149-155.

Anthonsen MW, Ronnstrand L, Wernstedt C, Degerman E \& Holm C (1998) Identification of novel phosphorylation sites in hormone-sensitive lipase that are phosphorylated in response to isoproterenol and govern activation properties in vitro. Journal of Biological Chemistry 273, 215-221.

Carlson LA, Ekelund LG \& Froberg SO (1971) Concentration of triglycerides phospholipids and glycogen in skeletal muscle and of free fatty acids and beta-hydroxybutyric acid in blood in man in response to exercise. European Journal of Clinical Investigation 1, 248-254.

Donsmark M, Langfort J, Holm C, Ploug T \& Galbo H (2003) Contractions activate hormone-sensitive lipase in rat muscle by protein kinase $\mathrm{C}$ and mitogen-activated protein kinase. Journal of Physiology (London) 550, 845-854.

Enevoldsen LH, Stallknecht B, Fluckey JD \& Galbo H (2000) Effect of exercise training on in vivo lipolysis in intraabdominal adipose tissue in rats. American Journal of Physiology 279, E585-E592.

Enevoldsen LH, Stallknecht B, Langfort J, Petersen LN, Holm C, Ploug T \& Galbo H (2001) The effect of exercise training on hormone-sensitive lipase in rat intra-abdominal adipose tissue and muscle. Journal of Physiology (London) 536, 871-877.

Fredrikson G, Tornqvist H \& Belfrage P (1986) Hormonesensitive lipase and monoacylglycerol lipase are both required for complete degradation of adipocyte triacylglycerol. Biochimica et Biophysica Acta 876, 288-293.

Galbo H (1983) Hormonal and Metabolic Adaptations to Exercise. Philadelphia, PA: WB Saunders Company. 
Garton AJ, Campbell DG, Carling D, Hardie DG, Colbran RJ \& Yeaman SJ (1989) Phosphorylation of bovine hormonesensitive lipase by the AMP-activated protein-kinase - A possible antilipolytic mechanism. European Journal of Biochemistry 179, 249-254.

Greenberg AS, Shen WJ, Muliro K, Patel S, Souza SC, Roth RA \& Kraemer FB (2001) Stimulation of lipolysis and hormonesensitive lipase via the extracellular signal-regulated kinase pathway. Journal of Biological Chemistry 276, 45456-45461.

Holm C, Belfrage P \& Fredrikson G (1987) Immunological evidence for the presence of hormone-sensitive lipase in rattissues other than adipose-tissue. Biochemical and Biophysical Research Communications 148, 99-105.

Holm C, Kirchgessner TG, Svenson KL, Fredrikson G, Nilsson S, Miller CG, Shively JE, Heinzmann C, Sparkes RS, Mohandas T, Lusis AJ, Belfrage P \& Schotz MC (1988) Hormonesensitive lipase - Sequence expression, and chromosomal localization to 19 Cent-Q13.3. Science 241, 1503-1506.

Holm C \& Østerlund T (1998) Hormone-sensitive lipase and cholesteryl esterlipase. In Methods in Molecular Biology: Lipases and Phospholipases Protocols, pp. 109-121 [MH Doolittle and K Reue, editors]. Totowa, NJ: Human Press Inc.

Holm C, Østerlund T, Laurell H \& Contreras JA (2000) Molecular mechanisms regulating hormone-sensitive lipase and lipolysis. Annual Review of Nutrition 20, 365-393.

Hopp JF \& Palmer WK (1990) Effect of electrical-stimulation on intracellular triacylglycerol in isolated skeletal-muscle. Journal of Applied Physiology 68, 348-354.

Hurley BF, Nemeth PM, Martin WH, Hagberg JM, Dalsky GP \& Holloszy JO (1986) Muscle triglyceride utilization during exercise - Effect of training. Journal of Applied Physiology 60, $562-567$.

Langfort J, Ploug T, Donsmark M, Gorski J \& Galbo H (2002) Training enhances contraction-mediated hormone-sensitive lipase activation in rat soleus muscle. In 7th Annual Congress of the European College of Sports Science, 24-28 July, Athens, p. 1137 [M Koskolou, N Geladas and V Klissouras, editors]. Athens, Greece: Pashalidis Medical Publisher.

Langfort J, Ploug T, Ihlemann J, Baranczuk E, Donsmark M, Gorski J \& Galbo H (2003) Additivity of adrenaline and contractions on hormone-sensitive lipase, but not on glycogen phosphorylase, in rat muscle. Acta Physiologica Scandinavica 178, 51-60.

Langfort J, Ploug T, Ihlemann J, Holm C \& Galbo H (2000) Stimulation of hormone-sensitive lipase activity by contractions in rat skeletal muscle. Biochemical Journal 351, 207-214.

Langfort J, Ploug T, Ihlemann J, Saldo M, Holm C \& Galbo H (1999) Expression of hormone-sensitive lipase and its regulation by adrenaline in skeletal muscle. Biochemical Journal 340, 459-465.

Langin D, Holm C \& Lafontan M (1996) Adipocyte hormonesensitive lipase: A major regulator of lipid metabolism. Proceedings of the Nutrition Society 55, 93-109.
Large V, Arner P, Reynisdottir S, Grober J, Van Harmelen V, Holm C \& Langin D (1998) Hormone-sensitive lipase expression and activity in relation to lipolysis in human fat cells. Journal of Lipid Research 39, 1688-1695.

Large V, Reynisdottir S, Langin D, Fredby K, Klannemark M, Holm C \& Arner P (1999) Decreased expression and function of adipocyte hormone-sensitive lipase in subcutaneous fat cells of obese subjects. Journal of Lipid Research 40, 2059-2066.

Martin WH III (1996) Effects of acute and chronic exercise on fat metabolism. Exercise and Sport Sciences Reviews 24, 203-231.

Napoli R, Gibson L, Hirshman MF, Boppart MD, Dufresne SD, Horton ES \& Goodyear LJ (1998) Epinephrine and insulin stimulate different mitogen-activated protein kinase signaling pathways in rat skeletal muscle. Diabetes 47, 1549-1554.

Oscai LB, Essig DA \& Palmer WK (1990) Lipase regulation of muscle triglyceride hydrolysis. Journal of Applied Physiology 69, 1571-1577.

Phillips SM, Green HJ, Tarnopolsky MA, Heigenhauser GJ \& Grant SM (1996) Progressive effect of endurance training on metabolic adaptations in working skeletal muscle. American Journal of Physiology 270, E265-E272.

Reitman J, Baldwin KM \& Holloszy JO (1973) Intramuscular triglyceride utilization by red, white, and intermediate skeletal muscle and heart during exhausting exercise. Proceedings of the Society for Experimental Biology and Medicine 142, 628-631.

Small CA, Garton AJ \& Yeaman SJ (1989) The presence and role of hormone-sensitive lipase in heart-muscle. Biochemical Journal 258, 67-72.

Spriet LL, Heigenhauser GJ \& Jones NL (1986) Endogenous triacylglycerol utilization by rat skeletal-muscle during tetanic stimulation. Journal of Applied Physiology 60, 410-415.

Straalfors P, Olsson H \& Belfrage P (1987) Hormone-sensitive lipase. In The Enzymes, vol. 18, pp. 147-177 [PD Boyer and EG Krebs, editors]. New York: Academic Press Inc.

Su CL, Sztalryd C, Contreras JA, Holm C, Kimmel AR \& Londos C (2003) Mutational analysis of the hormone-sensitive lipase translocation reaction in adipocytes. Journal of Biological Chemistry 278, 43615-43619.

Sztalryd C \& Kraemer FB (1994) Regulation of hormonesensitive lipase during fasting. American Journal of Physiology 266, E179-E185.

Van der Vusse GJ \& Reneman RS (1996) Exercise: Regulation and Integration of Multiple Systems, pp. 952-994. Oxford: Oxford University Press.

Vaughan M, Berger JE \& Steinberg D (1964) Hormone-sensitive lipase and monoglyceride lipase activities in adipose tissue. Journal of Biological Chemistry 239, 401-409.

Widegren U, Ryder JW \& Zierath JR (2001) Mitogen-activated protein kinase signal transduction in skeletal muscle: effects of exercise and muscle contraction. Acta Physiologica Scandinavica 172, 227-238.

Yeaman SJ (1990) Hormone-sensitive lipase - A multipurpose enzyme in lipid-metabolism. Biochimica et Biophysica Acta 1052, 128-132. 